\title{
Fast Learning Requires Good Memory: A Time-Space Lower Bound for Parity Learning
}

\author{
Ran Raz*
}

\begin{abstract}
We prove that any algorithm for learning parities requires either a memory of quadratic size or an exponential number of samples. This proves a recent conjecture of Steinhardt, Valiant and Wager SVW15] and shows that for some learning problems a large storage space is crucial.

More formally, in the problem of parity learning, an unknown string $x \in\{0,1\}^{n}$ was chosen uniformly at random. A learner tries to learn $x$ from a stream of samples $\left(a_{1}, b_{1}\right),\left(a_{2}, b_{2}\right) \ldots$, where each $a_{t}$ is uniformly distributed over $\{0,1\}^{n}$ and $b_{t}$ is the inner product of $a_{t}$ and $x$, modulo 2 . We show that any algorithm for parity learning, that uses less than $\frac{n^{2}}{25}$ bits of memory, requires an exponential number of samples.

Previously, there was no non-trivial lower bound on the number of samples needed, for any learning problem, even if the allowed memory size is $O(n)$ (where $n$ is the space needed to store one sample).

We also give an application of our result in the field of bounded-storage cryptography. We show an encryption scheme that requires a private key of length $n$, as well as time complexity of $n$ per encryption/decription of each bit, and is provenly and unconditionally secure as long as the attacker uses less than $\frac{n^{2}}{25}$ memory bits and the scheme is used at most an exponential number of times. Previous works on boundedstorage cryptography assumed that the memory size used by the attacker is at most linear in the time needed for encryption/decription.
\end{abstract}

\footnotetext{
${ }^{*}$ Weizmann Institute of Science, Israel, and the Institute for Advanced Study, Princeton, NJ. Research supported by the Israel Science Foundation grant No. 1402/14, by the I-CORE Program of the Planning and Budgeting Committee and the Israel Science Foundation, by the Simons Collaboration on Algorithms and Geometry, by the Fund for Math at IAS, and by the National Science Foundation grant No. CCF1412958. Any opinions, findings and conclusions or recommendations expressed in this material are those of the author and do not necessarily reflect the views of the National Science Foundation. Email: ran.raz.mail@gmail.com
} 


\section{Introduction}

Parity learning can be solved in polynomial time, by Gaussian elimination, using $O(n)$ samples and $O\left(n^{2}\right)$ memory bits. On the other hand, parity learning can be solved by trying all the possibilities, using $n+o(n)$ memory bits and an exponential number of samples.

We prove that any algorithm for parity learning requires either $\frac{n^{2}}{25}$ memory bits, or an exponential number of samples. Our result may be of interest from the points of view of learning theory, computational complexity and cryptography.

\section{$1.1 \quad$ Learning Theory}

The main message of this paper from the point of view of learning theory is that for some learning problems, access to a relatively large memory is crucial. In other words, in some cases, learning is infeasible, due to memory constraints. We show that there exist concept classes that can be efficiently learnt from a polynomial number of samples, if the learner has access to a quadratic-size memory, but require an exponential number of samples if the memory used by the learner is of less than quadratic size. This gives a formally stated and mathematically proved example for the intuitive feeling that a "good" memory may be very helpful in learning processes.

Many works studied the resources needed for learning, under certain information, communication or memory constraints (see in particular [514, 5VW15] and the many references given there). However, there was no previous non-trivial lower bound on the number of samples needed, for any learning problem, even when the allowed memory size is bounded by the length of one sample (where we don't count the space taken by the current sample that is being read).

The starting point of our work is the intriguing recent work of Steinhardt, Valiant and Wager [SVW15]. Steinhardt, Valiant and Wager asked whether there exist concept classes that can be efficiently learnt from a polynomial number of samples, but cannot be learnt from a polynomial number of samples if the allowed memory size is linear in the length of one sample. They conjectured that the problem of parity learning provides such a separation. Our main result proves that conjecture.

Remark 1.1. Conjecture 1.1 of SVW13 conjectures that any algorithm for parity learning requires either at least $\frac{n^{2}}{4}$ bits of memory, or at least $2^{n / 4}$ samples. Our main result qualitatively proves this conjecture, but with different constants. The conjecture, as stated, (that is, with the ambitious constants $\frac{1}{4}, \frac{1}{4}$ ) is too strong.

\footnotetext{
${ }^{1}$ Roughly speaking, this is the case since an algorithm similar to Gaussian elimination can solve parity learning, using $\frac{n^{2}}{4}+O(n)$ memory bits and a polynomial number of samples (by keeping in step $k$, a matrix with $k$ rows and $n$ columns, where the first $k$ columns form the identity matrix). If $2^{n / 4}$ samples are available, one can essentially solve a parity learning problem of size $\frac{3}{4} n+o(n)$, by considering only samples with coefficients 0 on the last $\frac{1}{4} n-o(n)$ variables. Hence, if $2^{n / 4}$ samples are available, $\frac{9}{64} n^{2}+o\left(n^{2}\right)$ memory bits are sufficient.
} 


\subsection{Computational Complexity}

Time-space tradeoffs have been extensively studied in the field of computational complexity, in many works and various settings. Two brilliant lines of research were particularly successful in establishing time-space lower bounds for computation.

The first line of works BJS98, A99a, A99b, BSSV00 gives explicit examples for polynomial-time computable Boolean functions $f:\{0,1\}^{n} \rightarrow\{0,1\}$, such that, any algorithm for computing $f$ requires either at least $n^{1-\epsilon}$ memory bits, where $\epsilon>0$ is an

arbitrarily small constant, or time complexity of at least $\Omega(n \sqrt{\log n / \log \log n})$. These bounds are proved for any branching program that computes $f$. Branching programs are the standard and most general computational model for studying time-space tradeoffs in the non-uniform setting (which is the more general setting), and is also the computational model that we use in the current work.

The second line of works F97, FLvMV05, W06, W07 (and other works) studies timespace tradeoffs for $S A T$ (and other NP problems), in the uniform setting, and proves that any algorithm for $S A T$ requires either at least $n^{1-\epsilon}$ memory bits, or time complexity of at least $n^{1+\delta}$ (where $0<\epsilon, \delta<1$ are constants). For an excellent survey, see [vM07].

Both lines of works obtain less than quadratic lower bounds on the time needed for computation, under memory constraints. Quadratic lower bounds on the time needed for computation are not known, even if the allowed memory-size is logarithmic. Comparing these results to our work, one may ask what makes it possible to prove exponential lower bounds on the time needed for parity learning, under memory constraints, while the known time-space lower bounds for computations are significantly weaker? The main point to keep in mind is that when studying time-space tradeoffs for computing a function, one assumes that the input for the function can always be accessed, and the space needed to store the input doesn't count as memory that is used by the algorithm. Thus, the input is stored for free. In our learning problem, it is assumed that after the learner saw a sample, the learner cannot access that sample again, unless the sample was stored in the learner's memory. The learner can always get a new sample that is "as good as the old one", but she cannot access the same sample that she saw before (without storing it in the memory).

Finally, let us note that by Barrington's celebrated result, any function in $N C$ can be computed by a polynomial-length branching program of width 5 [B86]. Hence, proving super-polynomial lower bounds on the time needed for computing a function, by a branching program of width 5, would imply super-polynomial lower bounds for formula size.

\subsection{Cryptography}

Assume that a group of (two or more) users share a (random) secret key $x \in\{0,1\}^{n}$. Assume that user Alice wants to send an encrypted bit $M \in\{0,1\}$ to user Bob. Let $a$ be a string of $n$ bits, uniformly distributed over $\{0,1\}^{n}$, and assume that both Alice and Bob know a (we can think of $a$ as taken from a shared random string and if a shared random string is not available Alice can just choose $a$ randomly and send it to Bob). Let $b$ be the inner product of $a$ and $x$, modulo 2. Thus, $b$ is known to both Alice and Bob and can be used as a one time pad to encrypt/decrypt $M$, that is, Alice encrypts by computing $M \oplus b$ and Bob decrypts by computing $M=(M \oplus b) \oplus b$. 
Assume that this protocol is used $m+1$ times, with the same secret key $x$, where $m$ is less than exponential. Denote by $a_{t}, b_{t}$ the string $a$ and bit $b$ used at time $t$. Suppose that during all that time, an attacker could see $\left(a_{1}, b_{1}\right), \ldots,\left(a_{m}, b_{m}\right)$, but the attacker has less than $\frac{n^{2}}{25}$ bits of memory. Our main result shows that the attacker cannot guess the secret key $x$, with better than exponentially small probability. Therefore, using the fact that inner product is a strong extractor (with exponentially small error), even if the attacker sees $a_{m+1}$, the attacker cannot predict $b_{m+1}$, with better than exponentially small advantage over a random guess.

Thus, if the attacker has less than $\frac{n^{2}}{25}$ bits of memory, the encryption remains secure as long as it is used less than an exponential number of times.

Bounded-storage cryptography, first introduced by Maurer M92 and extensively studied in many works, studies cryptographical protocols that are secure under the assumption that the memory used by the attacker is limited (see for example [CM97, AR99, ADR02, V03, DM04, and many other works). Previous works on bounded-storage cryptography assumed the existence of a high-rate source of randomness that streams random bits to all parties. The main idea is that the attacker doesn't have sufficiently large memory to store all random bits, and hence a shared secret key can be used to randomly select (or extract) bits from the random source that the attacker has very little information about.

In previous works, the number of random bits transmitted during the encryption was assumed to be larger than the memory-size of the attacker. Thus, the time needed for encryption/decryption was at least linear in the memory-size of the attacker. In contrast, the time needed for encryption/decryption in our protocol is $n$, while the encryption is secure against attackers with memory of size $\frac{n^{2}}{25}$.

Remark 1.2. If Alice and Bob want to transmit encrypted messages of length $m$, where $m \geq n$ (and the attacker has $O\left(n^{2}\right)$ bits of memory), our protocol has no advantage over previous ones, as the time needed for encryption/decription in our protocol is mn. The advantage of our protocol is in situations where the users want to securely transmit many shorter messages.

\subsection{Our Result}

\section{Parity Learning}

In the problem of parity learning, there is an unknown string $x \in\{0,1\}^{n}$ that was chosen uniformly at random. A learner tries to learn $x$ from samples $(a, b)$, where $a \in_{R}\{0,1\}^{n}$ and $b=a \cdot x$ (where $a \cdot x$ denotes inner product modulo 2). That is, the learning algorithm is given a stream of samples, $\left(a_{1}, b_{1}\right),\left(a_{2}, b_{2}\right) \ldots$, where each $a_{t}$ is uniformly distributed over $\{0,1\}^{n}$ and for every $t, b_{t}=a_{t} \cdot x$.

\section{Main Result}

Theorem 1. For any $c<\frac{1}{20}$, there exists $\alpha>0$, such that the following holds: Let $x$ be uniformly distributed over $\{0,1\}^{n}$. Let $m \leq 2^{\alpha n}$. Let $A$ be an algorithm that is given as input a stream of samples, $\left(a_{1}, b_{1}\right), \ldots,\left(a_{m}, b_{m}\right)$, where each $a_{t}$ is uniformly distributed over $\{0,1\}^{n}$ and for every $t, b_{t}=a_{t} \cdot x$. Assume that $A$ uses at most cn ${ }^{2}$ memory bits and outputs a string $\tilde{x} \in\{0,1\}^{n}$. Then, $\operatorname{Pr}[\tilde{x}=x] \leq O\left(2^{-\alpha n}\right)$. 
Theorem 11 is restated, in a strongerf and more formal 9 form, as Theorem 2 in Section 7 , and the proof of Theorem 2 is given there.

\section{Preliminaries}

For an integer $n$, denote $[n]=\{1, \ldots, n\}$. For $a, x \in\{0,1\}^{n}$, denote by $a \cdot x$ their inner product modulo 2 .

For a function $P: \Omega \rightarrow \mathbb{R}$, we denote by $|P|_{1}$ its $\ell_{1}$ norm. In particular, for two distributions, $P, Q: \Omega \rightarrow[0,1]$, we denote by $|P-Q|_{1}$ their $\ell_{1}$ distance.

For a random variable $X$ and an event $E$, we denote by $\mathbb{P}_{X}$ the distribution of the random variables $X$, and we denote by $\mathbb{P}_{X \mid E}$ the distribution of the random variable $X$ conditioned on the event $E$.

Denote by $\mathcal{U}_{n}$ the uniform distribution over $\{0,1\}^{n}$. For an affine subspace $w \subseteq\{0,1\}^{n}$, denote by $\mathcal{U}_{w}$ the uniform distribution over $w$.

For $n \in \mathbb{N}$, denote by $\mathcal{A}(n)$ the set of all affine subspaces of $\{0,1\}^{n}$.

\section{Proof Outline}

\section{Computational Model}

We model the learning algorithm by a branching program. A branching program of length $m$ and width $d$, for parity learning, is a directed (multi) graph with vertices arranged in $m+1$ layers containing at most $d$ vertices each. Intuitively, each layer represents a time step and each vertex represents a memory state of the learner. In the first layer, that we think of as layer 0 , there is only one vertex, called the start vertex. A vertex of outdegree 0 is called a leaf. Every non-leaf vertex in the program has $2^{n+1}$ outgoing edges, labeled by elements $(a, b) \in\{0,1\}^{n} \times\{0,1\}$, with exactly one edge labeled by each such $(a, b)$, and all these edges going into vertices in the next layer. Intuitively, these edges represent the action when reading $\left(a_{t}, b_{t}\right)$. The samples $\left(a_{1}, b_{1}\right), \ldots,\left(a_{m}, b_{m}\right) \in\{0,1\}^{n} \times\{0,1\}$ that are given as input, define a computation-path in the branching program, by starting from the start vertex and following at Step $t$ the edge labeled by $\left(a_{t}, b_{t}\right)$, until reaching a leaf.

Each leaf $v$ in the program is labeled by an affine subspace $w(v) \in \mathcal{A}(n)$, that we think of as the output of the program on that leaf. The program outputs the label $w(v)$ of the leaf $v$ reached by the computation-path. We interpret the output of the program as a guess that $x \in w(v)$.

We also consider affine branching programs, where every vertex $v$ (not necessarily a leaf) is labeled by an affine subspace $w(v) \in \mathcal{A}(n)$, such that, the start vertex is labeled by the space $\{0,1\}^{n} \in \mathcal{A}(n)$, and for any edge $(u, v)$, labeled by $(a, b)$, we have $w(u) \cap\left\{x^{\prime} \in\{0,1\}^{n}: a \cdot x^{\prime}=b\right\} \subseteq w(v)$. These properties guarantee that if the computation-

\footnotetext{
${ }^{2}$ Theorem 2 allows the algorithm to output an affine subspace of dimension $\leq \frac{3}{5} n$, and bounds by $2^{-\alpha n}$ the probability that $x$ belongs to that affine subspace.

${ }^{3}$ Theorem 2 models the algorithm by a branching program, which is more formal and clarifies that the theorem holds also in the (more general) non-uniform setting.
} 
path reaches a vertex $v$ then $x \in w(v)$. Thus, we can interpret $w(v)$ as an affine subspace that is known to contain $x$.

An affine branching program is called accurate if for (almost) all vertices $v$, the distribution of $x$, conditioned on the event that the computation-path reached $v$, is close to the uniform distribution over $w(v)$.

For exact definitions, see Section 5 .

\section{The High-Level Approach}

The proof has two parts. We prove lower bounds for affine branching programs, and we reduce general branching programs to affine branching programs. The hard part is the reduction from general branching programs to affine branching programs. We note that this reduction is very wasteful and expands the width of the branching program by a factor of

$2^{\Theta\left(n^{2}\right)}$. Nevertheless, since we allow our branching program to be of width up to $2^{O\left(n^{2}\right)}$, this is still affordable (as long as the exact constant in the exponent is relatively small). We have to make sure though that, when proving time-space lower bounds for affine branching programs, the upper bounds that we assume on the width of the affine branching programs are larger than the expansion of the width caused by the reduction.

We note that in the introduction to Conjecture 1.1 of SVW15, Steinhardt, Valiant and Wager mention that they were able to prove the conjecture "for any algorithm whose memory states correspond to subspaces". However, a formal statement (or proof) is not given, so we do not know how similar their result is to our lower bound for affine branching programs. We note that affine branching programs, as we define here, do not satisfy Conjecture 1.1 of [SVW15] (see Remark 1.1 and Footnote 1).

\section{Lower Bounds for Affine Branching Programs}

Assume that we have an affine branching program of length at most $2^{c n}$ and width at most $2^{c n^{2}}$, for a small enough constant $c$. Fix $k=\frac{4}{5} n$. We prove that the probability that the computation-path reaches some vertex that is labeled with an affine subspace of dimension $\leq k$ is at most $2^{-\Omega\left(n^{2}\right)}$.

Without loss of generality, we can assume that all vertices in the program are labeled with affine subspaces of dimension $\geq k$. Other vertices can just be removed as the computationpath must reach a vertex labeled with a subspace of dimension $k$, before it reaches a vertex labeled with a subspace of dimension $<k$ (because the dimension can decrease by at most 1 along an edge).

We define the "orthogonal" to an affine subspace as the vector space orthogonal to the vector space that defines that affine subspace (that is, the vector space that the affine subspace is given as it's translation).

Let $v$ be a vertex in the program, such that, $w(v)$ is of dimension $k$. It's enough to prove that the probability that the computation-path reaches $v$ is at most $2^{-\Omega\left(n^{2}\right)}$.

To prove this, we consider the vector spaces "orthogonal" to the affine subspaces that label the vertices along the computation-path, and for each of them we consider its intersection with the vector space "orthogonal" to $w(v)$. We note that, in each step, the probability that the dimension of the intersection increases is exponentially small (as it requires that the $a_{t}$ 
currently being read is contained in some small vector space). Since the dimension of the intersection must increase a linear number of times, in order for the computation-path to reach $v$, a simple union bound shows that the probability to reach $v$ is at most $2^{-\Omega\left(n^{2}\right)}$.

The full details are given in Lemma 7.1.

\section{From Branching Programs to Affine Branching Programs}

In Section 6, we show how to simulate a branching program by an accurate affine branching program. We do that layer after layer. Assume that we are already done with layer $j-1$, so every vertex in layer $j-1$ is already labeled by an affine subspace, and the distribution of $x$, conditioned on the event that the computation-path reached a vertex, is close to the uniform distribution over the affine subspace that labels that vertex.

Now, take a vertex $v$ in layer $j$, and consider the distribution of $x$, conditioned on the event that the computation-path reached the vertex $v$. By the property that we already know on layer $j-1$, this distribution is close to a convex combination of uniform distributions over affine subspaces of $\{0,1\}^{n}$.

One could split $v$ into a large number of vertices, one vertex for each affine subspace in the combination. However, this practically means that we would have a vertex for any affine subspace. We would like to keep the number of vertices somewhat smaller. This is done by grouping many affine subspaces into one group. The group will be labeled by an affine subspace that contains all the affine subspaces in the group. Moreover, we will have the property that for each such group, the uniform distribution over the affine subspace that labels the group is close to the relevant weighted average of the uniform distributions over the affine subspaces in the group. Thus, practically, we can replace all the affine subspaces in the group by one affine subspace that represents all of them.

Lemma 4.3 shows that it is possible to group all the affine subspaces into a relatively small number of groups.

We note that the entire inductive argument is delicate, as we cannot afford deteriorating the error multiplicatively in each step and need to make sure that all errors are additive.

\section{Distributions over Affine Subspaces}

In this section, we study convex combinations of uniform distributions over affine subspaces of $\{0,1\}^{n}$. Lemma 4.3 is the only result, proved in this section, that is used outside the section.

In the following lemmas, we have a random variable $W \in \mathcal{A}(n)$ and we consider the distribution $\mathbf{E}_{W}\left[\mathcal{U}_{W}\right]$. This distribution is a convex combination of uniform distributions over affine subspaces of $\{0,1\}^{n}$.

The first lemma identifies a condition that implies that the distribution $\mathbf{E}_{W}\left[\mathcal{U}_{W}\right]$ is close to the uniform distribution over $\{0,1\}^{n}$.

Lemma 4.1. Let $W \in \mathcal{A}(n)$ be a random variable. Let $r \geq \frac{n}{2}$. Assume that for every $a \in\{0,1\}^{n}$, such that $a \neq \overrightarrow{0}$, and every $b \in\{0,1\}$,

$$
\underset{W}{\operatorname{Pr}}[\forall x \in W: a \cdot x=b] \leq 2^{-r} .
$$


Then

$$
\left|\underset{W}{\mathbf{E}}\left[\mathcal{U}_{W}\right]-\mathcal{U}_{n}\right|_{1}<2^{-\left(r-\frac{n}{2}\right)}
$$

Proof. The proof uses Fourier analysis. For any affine subspace $w \subseteq\{0,1\}^{n}$, the Fourier coefficients of $\mathcal{U}_{w}$ are:

$$
\widehat{\mathcal{U}_{w}}(a)=\left\{\begin{array}{cc}
2^{-n} & \text { if } \forall x \in w: a \cdot x=0 \\
-2^{-n} & \text { if } \forall x \in w: a \cdot x=1 \\
0 & \text { otherwise }
\end{array}\right.
$$

Hence, the Fourier coefficients of $\mathbf{E}_{W}\left[\mathcal{U}_{W}\right]$ are:

$$
\widehat{\mathbf{E}\left[\mathcal{U}_{W}\right]}(a)=2^{-n} \cdot(\underset{W}{\operatorname{Pr}}[\forall x \in W: a \cdot x=0]-\underset{W}{\operatorname{Pr}}[\forall x \in W: a \cdot x=1]),
$$

and note that this also implies

$$
\widehat{\mathbf{E}\left[\mathcal{U}_{W}\right]}(\overrightarrow{0})=2^{-n}
$$

The Fourier coefficients of $\mathcal{U}_{n}$ are:

$$
\widehat{\mathcal{U}_{n}}(a)=\left\{\begin{array}{cl}
2^{-n} & \text { if } a=\overrightarrow{0} \\
0 & \text { if } a \neq \overrightarrow{0}
\end{array}\right.
$$

Thus,

$$
\sum_{a \in\{0,1\}^{n}}\left(\widehat{\mathbf{E}\left[\mathcal{U}_{W}\right]}(a)-\widehat{\mathcal{U}_{n}}(a)\right)^{2}<2^{n} \cdot\left(2^{-n} \cdot 2^{-r}\right)^{2}=2^{-n-2 r} .
$$

By Cauchy-Schwarz and Parseval,

$$
\begin{gathered}
\left(\underset{x \in R}{\mathbf{E}\{0,1\}^{n}}\left|\underset{W}{\mathbf{E}}\left[\mathcal{U}_{W}\right](x)-\mathcal{U}_{n}(x)\right|\right)^{2} \leq \underset{x \in \in_{R}\{0,1\}^{n}}{\mathbf{E}}\left(\underset{W}{\mathbf{E}}\left[\mathcal{U}_{W}\right](x)-\mathcal{U}_{n}(x)\right)^{2}= \\
\sum_{a \in\{0,1\}^{n}}\left(\widehat{\mathbf{E}\left[\mathcal{U}_{W}\right]}(a)-\widehat{\mathcal{U}_{n}}(a)\right)^{2}<2^{-n-2 r} .
\end{gathered}
$$

Therefore,

$$
\left|\underset{W}{\mathbf{E}}\left[\mathcal{U}_{W}\right]-\mathcal{U}_{n}\right|_{1}=2^{n} \underset{x \in R}{\mathbf{E}\{0,1\}^{n}}\left|\underset{W}{\mathbf{E}}\left[\mathcal{U}_{W}\right](x)-\mathcal{U}_{n}(x)\right|<2^{n} \cdot \sqrt{2^{-n-2 r}}=2^{-(r-n / 2)} .
$$

The next lemma shows that always there exists an affine subspace $s \subseteq\{0,1\}^{n}$, such that the distribution $\mathbf{E}_{W \mid(W \subseteq s)}\left[\mathcal{U}_{W}\right]$ is close to the uniform distribution over $s$, and the event $W \subseteq s$ occurs with non-negligible probability.

Lemma 4.2. Let $W \in \mathcal{A}(n)$ be a random variable. Let $r \geq \frac{n}{2}$. There exists an affine subspace $s \subseteq\{0,1\}^{n}$, such that:

1.

$$
\underset{W}{\operatorname{Pr}}[W \subseteq s] \geq 2^{-\sum_{i=0}^{n-\operatorname{dim}(s)-1}\left(r-\frac{i}{2}\right)} .
$$


2.

$$
\left|\underset{W \mid(W \subseteq s)}{\mathbf{E}}\left[\mathcal{U}_{W}\right]-\mathcal{U}_{s}\right|_{1}<2^{-\left(r-\frac{n}{2}\right)} .
$$

Proof. The proof is by induction on $n$. The base case, $n=0$, is trivial, because in this case the only element of $\mathcal{A}(n)$ is $\{\overrightarrow{0}\}$, so the lemma follows with $s=\{\overrightarrow{0}\}$.

Let $n \geq 1$. If for every $a \in\{0,1\}^{n}$, such that $a \neq \overrightarrow{0}$, and every $b \in\{0,1\}$, we have $\operatorname{Pr}_{W}[\forall x \in W: a \cdot x=b] \leq 2^{-r}$, the proof follows by Lemma 4.1, with $s=\{0,1\}^{n}$. Otherwise, there exists $a \neq \overrightarrow{0}$, and $b \in\{0,1\}$, such that, $\operatorname{Pr}_{W}[\forall x \in W: a \cdot x=b]>2^{-r}$. Denote by $u$ the $(n-1)$-dimensional affine subspace

$$
u=\left\{x \in\{0,1\}^{n}: a \cdot x=b\right\} .
$$

Thus,

$$
\underset{W}{\operatorname{Pr}}[W \subseteq u]>2^{-r}
$$

Consider the random variable $W^{\prime}=W \mid(W \subseteq u)$. Since $u$ is an $(n-1)$-dimensional affine subspace, we can identify $u$ with $\{0,1\}^{n-1}$ and think of $W^{\prime}$ as a random variable over $\mathcal{A}(n-1)$. Hence, by the inductive hypothesis (applied with $n-1$ and $r-\frac{1}{2}$ ), there exists an affine subspace $s \subseteq u$, such that,

1.

$$
\underset{W^{\prime}}{\operatorname{Pr}}\left[W^{\prime} \subseteq s\right] \geq 2^{-\sum_{i=1}^{n-\operatorname{dim}(s)-1}\left(r-\frac{i}{2}\right)}
$$

2.

$$
\left|\underset{W^{\prime} \mid\left(W^{\prime} \subseteq s\right)}{\mathbf{E}}\left[\mathcal{U}_{W^{\prime}}\right]-\mathcal{U}_{s}\right|_{1}<2^{-\left(r-\frac{n}{2}\right)} .
$$

We will show that $s$ satisfies the two properties claimed in the statement of the lemma.

For the first property, note that since $s \subseteq u$,

$$
\begin{aligned}
\operatorname{Pr}[W \subseteq s]= & \operatorname{Pr}[W \subseteq u] \cdot \operatorname{Pr}[W \subseteq s \mid W \subseteq u]=\operatorname{Pr}[W \subseteq u] \cdot \operatorname{Pr}\left[W^{\prime} \subseteq s\right] \\
& >2^{-r} \cdot 2^{-\sum_{i=1}^{n-\operatorname{dim}(s)-1}\left(r-\frac{i}{2}\right)}=2^{-\sum_{i=0}^{n-\operatorname{dim}(s)-1}\left(r-\frac{i}{2}\right)}
\end{aligned}
$$

For the second property, note that since $s \subseteq u$,

$$
\underset{W \mid(W \subseteq s)}{\mathbf{E}}\left[\mathcal{U}_{W}\right]=\underset{W^{\prime} \mid\left(W^{\prime} \subseteq s\right)}{\mathbf{E}}\left[\mathcal{U}_{W^{\prime}}\right]
$$

The next lemma is the main result of this section.

Lemma 4.3. Let $W \in \mathcal{A}(n)$ be a random variable. Let $r \geq \frac{n}{2}$. There exists a partial function $\sigma: \mathcal{A}(n) \rightarrow \mathcal{A}(n)$, such that:

1. $\operatorname{Pr}_{W}[W \notin \operatorname{domain}(\sigma)] \leq 2^{-2 n}$.

2. For every $w \in \operatorname{domain}(\sigma), w \subseteq \sigma(w)$. 
3. For every $s \in$ image $(\sigma)$,

$$
\left|\underset{W \mid(\sigma(W)=s)}{\mathbf{E}}\left[\mathcal{U}_{W}\right]-\mathcal{U}_{s}\right|_{1}<2^{-\left(r-\frac{n}{2}\right)} .
$$

4. For every $k \in \mathbb{N}$, there are at most

$$
4 n \cdot 2^{\sum_{i=0}^{n-k-1}\left(r-\frac{i}{2}\right)}
$$

elements $s \in \operatorname{image}(\sigma)$, with $\operatorname{dim}(s) \geq k$.

Proof. The proof is by repeatedly applying Lemma 4.2. We start with the random variable $W_{0}=W$, and apply Lemma 4.2 on $W_{0}$. We obtain a subspace $s_{0}$ (the subspace $s$ whose existence is guaranteed by Lemma 1.2). For every $w \subseteq s_{0}$, we define $\sigma(w)=s_{0}$.

We then define the random variable $W_{1}=W_{0} \mid\left(W_{0} \nsubseteq s_{0}\right)$, and apply Lemma 4.2 on $W_{1}$. We obtain a subspace $s_{1}$ (the subspace $s$ whose existence is guaranteed by Lemma 4.2). For every $w \subseteq s_{1}$ on which $\sigma$ was still not defined, we define $\sigma(w)=s_{1}$.

In the same way, in Step $i$, we define the random variable $W_{i}=W_{i-1} \mid\left(W_{i-1} \nsubseteq s_{i-1}\right)$. Note that $W_{i}=W \mid\left(W \nsubseteq s_{0}\right) \wedge \ldots \wedge\left(W \nsubseteq s_{i-1}\right)$, that is, $W_{i}$ is the restriction of $W$ to the part of $\mathcal{A}(n)$ where $\sigma$ was still not defined. We apply Lemma 1.2 on $W_{i}$ and obtain a subspace $s_{i}$ (the subspace $s$ whose existence is guaranteed by Lemma 4.2). For every $w \subseteq s_{i}$ on which $\sigma$ was still not defined, we define $\sigma(w)=s_{i}$.

We repeat this until $\operatorname{Pr}_{W}[W \notin \operatorname{domain}(\sigma)] \leq 2^{-2 n}$.

Note that for $i^{\prime}<i, s_{i^{\prime}} \neq s_{i}$, because the support of $W_{i}$ doesn't contain any element $w \subseteq s_{i^{\prime}}$. Hence, the subspaces $s_{0}, s_{1}, \ldots$ are all different.

It remains to show that the four properties in the statement of the lemma hold.

The first property is obvious because we continue to define $\sigma$ on more and more elements repeatedly, until the first property holds.

The second property is obvious because we mapped $w$ to $s_{i}$ only if $w \subseteq s_{i}$.

The third property holds by the second property guaranteed by Lemma 1.2 .

The forth property holds because by the first property guaranteed by Lemma 4.2, in each step where we obtain a subspace $s_{i}$ of dimension at least $k$, we define $\sigma$ on a fraction of at least $2^{-\sum_{i=0}^{n-k-1}\left(r-\frac{i}{2}\right)}$ of the space that still remains. Thus, after at most $4 n \cdot 2^{\sum_{i=0}^{n-k-1}\left(r-\frac{i}{2}\right)}$ such steps we have $\operatorname{Pr}[W \notin$ domain $(\sigma)] \leq 2^{-2 n}$, and we stop. Thus, the number of elements $s_{i}$, of dimension at least $k$, that we obtain in the process, is at most $4 n \cdot 2^{\sum_{i=0}^{n-k-1}\left(r-\frac{i}{2}\right)}$.

\section{Branching Programs for Parity Learning}

Recall that in the problem of parity learning, there is a string $x \in\{0,1\}^{n}$ that was chosen uniformly at random. A learner tries to learn $x$ from a stream of samples, $\left(a_{1}, b_{1}\right),\left(a_{2}, b_{2}\right) \ldots$, where each $a_{t}$ is uniformly distributed over $\{0,1\}^{n}$ and for every $t, b_{t}=a_{t} \cdot x$.

\subsection{General Branching Programs for Parity Learning}

In the following definition, we model the learner by a branching program. We allow the branching program to output an affine subspace $w \in \mathcal{A}(n)$. We interpret the output of the 
program as a guess that $x \in w$. Obviously, the output $w$ is more meaningful when $\operatorname{dim}(w)$ is relatively small.

Definition 5.1. Branching Program for Parity Learning: A branching program of length $m$ and width $d$, for parity learning, is a directed (multi) graph with vertices arranged in $m+1$ layers containing at most $d$ vertices each. In the first layer, that we think of as layer 0 , there is only one vertex, called the start vertex. A vertex of outdegree 0 is called a leaf. All vertices in the last layer are leaves (but there may be additional leaves). Every non-leaf vertex in the program has $2^{n+1}$ outgoing edges, labeled by elements $(a, b) \in\{0,1\}^{n} \times\{0,1\}$, with exactly one edge labeled by each such $(a, b)$, and all these edges going into vertices in the next layer. Each leaf $v$ in the program is labeled by an affine subspace $w(v) \in \mathcal{A}(n)$, that we think of as the output of the program on that leaf.

Computation-Path: The samples $\left(a_{1}, b_{1}\right), \ldots,\left(a_{m}, b_{m}\right) \in\{0,1\}^{n} \times\{0,1\}$ that are given as input, define a computation-path in the branching program, by starting from the start vertex and following at Step $t$ the edge labeled by $\left(a_{t}, b_{t}\right)$, until reaching a leaf. The program outputs the label $w(v)$ of the leaf $v$ reached by the computation-path.

Success Probability: The success probability of the program is the probability that $x \in w$, where $w$ is the affine subspace that the program outputs, and the probability is over $x, a_{1}, \ldots, a_{m}$ (where $x, a_{1}, \ldots, a_{m}$ are uniformly distributed over $\{0,1\}^{n}$, and for every $t$, $\left.b_{t}=a_{t} \cdot x\right)$.

\subsection{Affine Branching Programs for Parity Learning}

Next, we define a special type of a branching program for parity learning, that we call an affine branching program for parity learning. In an affine branching program for parity learning, every vertex $v$ (not necessarily a leaf) is labeled by an affine subspace $w(v) \in \mathcal{A}(n)$. We will have the property that if the computation-path reaches $v$ then $x \in w(v)$. Thus, we can interpret $w(v)$ as an affine subspace that is known to contain $x$.

Definition 5.2. Affine Branching Program for Parity Learning: A branching program for parity learning is affine if each vertex $v$ in the program is labeled by an affine subspace $w(v) \in \mathcal{A}(n)$, and the following properties hold:

1. Start vertex: The start vertex is labeled by the space $\{0,1\}^{n} \in \mathcal{A}(n)$.

2. Soundness: For an edge $e=(u, v)$, labeled by $(a, b)$, denote

$$
w(e)=w(u) \cap\left\{x^{\prime} \in\{0,1\}^{n}: a \cdot x^{\prime}=b\right\} .
$$

Then,

$$
w(e) \subseteq w(v)
$$

Given an affine branching program for parity learning, and samples $\left(a_{1}, b_{1}\right), \ldots,\left(a_{m}, b_{m}\right)$, such that, for every $t, b_{t}=a_{t} \cdot x$, it follows by induction that for every vertex $v$ in the program, if the computation-path reaches $v$ then $x \in w(v)$. In particular, the output $w$ of the program always satisfies $x \in w$, and thus the success probability of an affine program is always 1. 


\subsection{Accurate Affine Branching Programs for Parity Learning}

For a vertex $v$ in a branching program for parity learning, we denote by $\mathbb{P}_{x \mid v}$ the distribution of the random variable $x$, conditioned on the event that the vertex $v$ was reached by the computation-path.

Definition 5.3. $\epsilon$-Accurate Affine Branching Program for Parity Learning: An affine branching program of length $m$ for parity learning is $\epsilon$-accurate if all the leaves are in the last layer, and the following additional property holds (where $x, a_{1}, \ldots, a_{m}$ are uniformly distributed over $\{0,1\}^{n}$, and for every $\left.t, b_{t}=a_{t} \cdot x\right)$ :

3. Accuracy: Let $0 \leq t \leq m$. Let $V_{t}$ be the vertex in layer $t$, reached by the computationpath. Let $y_{t}$ be a random variable uniformly distributed over the subspace $w\left(V_{t}\right)$, Then,

$$
\left|\mathbb{P}_{V_{t}, x}-\mathbb{P}_{V_{t}, y_{t}}\right|_{1} \leq \epsilon
$$

or, equivalently,

$$
\underset{V_{t}}{\mathbf{E}}\left|\mathbb{P}_{x \mid V_{t}}-\mathcal{U}_{w\left(V_{t}\right)}\right|_{1} \leq \epsilon
$$

\section{From Branching Programs to Affine Branching Programs}

In this section, we show that any branching program $B$ for parity learning can be simulated by an affine branching program $P$ for parity learning. Roughly speaking, each vertex of the simulated program $B$ will be represented by a set of vertices of the simulating program $P$. Note that the width of $P$ will typically be significantly larger than the width of $B$.

More precisely, a branching program $B$ for parity learning is simulated by a branching program $P$ for parity learning if there exists a mapping $\Gamma$ from the vertices of $P$ to the vertices of $B$, and the following properties hold:

1. Preservation of structure: For every $i, \Gamma$ maps layer $i$ of $P$ to layer $i$ of $B$. Moreover, $\Gamma$ maps leaves to leaves and non-leaf vertices to non-leaf vertices. Note that $\Gamma$ is not necessarily one-to-one.

2. Preservation of functionality: For every edge $(u, v)$, labeled by $(a, b)$, in $P$, there is an edge $(\Gamma(u), \Gamma(v))$, labeled by $(a, b)$, in $B$.

Lemma 6.1. Let $k^{\prime}<n$. Assume that there exists a length $m$ and width d branching program $B$ for parity learning (of size $n$ ), such that: all leaves of $B$ are in the last layer; the output of $B$ is always an affine subspace of dimension $\leq k^{\prime}$; and the success probability of $B$ is $\beta$.

Let $\frac{n}{2} \leq r \leq n$. Let $\epsilon=4 m \cdot 2^{-\left(r-\frac{n}{2}\right)}$. Then, there exists an $\epsilon$-accurate length $m$ affine branching program $P$ for parity learning (of size $n$ ), such that:

1. For every $k<n$, the number of vertices in $P$, that are labeled with an affine subspace of dimension $k$, is at most

$$
4 n \cdot 2^{\sum_{i=0}^{n-k-1}\left(r-\frac{i}{2}\right)} \cdot d m
$$


2. For every $k$, such that, $k^{\prime}<k<n$, the output of $P$ is an affine subspace of dimension $<k$, with probability of at least

$$
\beta-\epsilon-2^{-\left(k-k^{\prime}\right)}
$$

Proof. For every $0 \leq j \leq m$, let $\epsilon_{j}=4 j \cdot 2^{-\left(r-\frac{n}{2}\right)}$. We will use Lemma 4.3 to turn, inductively, the layers of $B$, one by one, into layers of an $\epsilon$-accurate affine branching program, $P$. In Step $j$ of the induction, we will turn layer $j$ of $B$ into layer $j$ of $P$, and define the label $w(v) \in \mathcal{A}(n)$ for every vertex $v$ in that layer of $P$. Formally, we will construct, inductively, a sequence of programs $B, P_{0}, \ldots, P_{m}=P$, where each program is of length $m$, and for every $j$, the program $P_{j}$ differs from the previous program only in layer $j$ (and in the edges going into layer $j$ and out of layer $j$ ). After Step $j$ of the induction, we will have a branching program $P_{j}$, such that, layers 0 to $j$ of $P_{j}$ form an affine branching program for parity learning. In addition, the following inductive hypothesis will hold:

\section{Inductive Hypothesis:}

Let $\mathcal{L}_{j}$ be the set of vertices in layer $j$ of $P_{j}$. Let $V_{j}$ be the vertex in $\mathcal{L}_{j}$, reached by the computation-path of $P_{j}$. Note that $V_{j}$ is a random variable that depends on $x, a_{1}, \ldots, a_{j}$ (and recall that $x, a_{1}, \ldots, a_{m}$ are uniformly distributed over $\{0,1\}^{n}$, and for every $\left.t, b_{t}=a_{t} \cdot x\right)$. The inductive hypothesis is that there exists a random variable $U_{j}$ over $\mathcal{L}_{j}$, such that, if $y_{j}$ is a random variable uniformly distributed over the subspace $w\left(U_{j}\right)$, then

$$
\left|\mathbb{P}_{V_{j}, x}-\mathbb{P}_{U_{j}, y_{j}}\right|_{1} \leq \frac{\epsilon_{j}}{2} .
$$

The inductive hypothesis is equivalent to the accuracy requirement (see Definition 5.3) for layer $j$ of $P_{j}$, up to a small multiplicative constant in the accuracy, but we need to assume it in this slightly different form, in order to avoid deteriorating the accuracy by a multiplicative factor in each step of the induction.

\section{Base Case:}

In the base case of the induction, $j=0$, we define $P_{0}$ by just labeling the start vertex of $B$ by $\{0,1\}^{n} \in \mathcal{A}(n)$. Thus, the start vertex property in the definition of an affine branching program is satisfied. The soundness property is trivially satisfied because the restriction of $P_{0}$ to layer 0 contains no edges. Since we always start from the start vertex, the distribution of the random variable $x$, conditioned on the event that we reached the start vertex, is just $\mathcal{U}_{n}$, and hence the inductive hypothesis (Equation (11) ) holds with $U_{0}=V_{0}$.

\section{Inductive Step:}

Assume that we already turned layers 0 to $j-1$ of $B$ into layers 0 to $j-1$ of $P$. That is, we already defined the program $P_{j-1}$, and layers 0 to $j-1$ of $P_{j-1}$ satisfy the start vertex property, the soundness property, and the inductive hypothesis (Equation (四)). We will now show how to define $P_{j}$ from $P_{j-1}$, that is, how to turn layer $j$ of $B$ into layer $j$ of $P$.

Let $U_{j-1} \in \mathcal{L}_{j-1}$ be the random variable that satisfies the inductive hypothesis (Equation (10) for layer $j-1$ of $P_{j-1}$. Let $y_{j-1}$ be a random variable uniformly distributed 
over the subspace $w\left(U_{j-1}\right)$. Let $a \in_{R}\{0,1\}^{n}$. Let $b=a \cdot y_{j-1}$. Let $E=\left(U_{j-1}, V\right)$ be the edge labeled by $(a, b)$ outgoing $U_{j-1}$ in $P_{j-1}$. Thus, $V$ is a vertex in layer $j$ of $P_{j-1}$. Let $W=w(E)$, where $w(E)$ is defined as in the soundness property in Definition 5.2. That is,

$$
w(E)=w\left(U_{j-1}\right) \cap\left\{x^{\prime} \in\{0,1\}^{n}: a \cdot x^{\prime}=b\right\},
$$

where $(a, b)$ is the label of $E$, and $w\left(U_{j-1}\right)$ is the label of $U_{j-1}$ in $P_{j-1}$.

Let $v$ be a vertex in layer $j$ of $P_{j-1}$ (and note that $v$ is also a vertex in layer $j$ of $B$ ). Let

$$
W_{v}=W \mid(V=v) .
$$

Let $\sigma_{v}: \mathcal{A}(n) \rightarrow \mathcal{A}(n)$ be the partial function whose existence is guaranteed by Lemma 4.3 , when applied on the random variable $W_{v}$. Extend $\sigma_{v}: \mathcal{A}(n) \rightarrow \mathcal{A}(n)$ so that it outputs the special value $*$ on every element where it was previously undefined.

In the program $P_{j}$, we will split the vertex $v$ into $\mid$ image $\left(\sigma_{v}\right) \mid \operatorname{vertices}\left(\right.$ where image $\left(\sigma_{v}\right)$ already contains the additional special value $*)$. For every $s \in \operatorname{image}\left(\sigma_{v}\right)$, we will have a vertex $(v, s)$. If $s \neq *$, we label the vertex $(v, s)$ by the affine subspace $s$, and we label the additional vertex $(v, *)$ by $\{0,1\}^{n}$. For every $s \in \operatorname{image}\left(\sigma_{v}\right)$, the edges going out of $(v, s)$ (in $P_{j}$ ) will be the same as the edges going out of $v$ in $P_{j-1}$. That is, for every edge $\left(v, v^{\prime}\right)$ (from layer $j$ to layer $j+1$ ) in the program $P_{j-1}$, and every $s \in \operatorname{image}\left(\sigma_{v}\right)$, we will have an edge $\left((v, s), v^{\prime}\right)$ with the same label, (from layer $j$ to layer $j+1$ ) in the program $P_{j}$.

We will now define the edges going into the vertices $(v, s)$ in the program $P_{j}$. For every edge $e=(u, v)$, labeled by $(a, b)$, (from layer $j-1$ to layer $j$ ), in the program $P_{j-1}$, consider the affine subspace $w=w(e)=w(u) \cap\left\{x^{\prime} \in\{0,1\}^{n}: a \cdot x^{\prime}=b\right\}$ (as in the soundness property in Definition 5.2), where $w(u)$ is the label of $u$ in $P_{j-1}$. Let $s=\sigma_{v}(w)$.

In $P_{j}$, we will have the edge $(u,(v, s)$ ) (labeled by $(a, b)$ ), from layer $j-1$ to layer $j$, that is, we connect $u$ to $(v, s)$. Note that the edge $(u,(v, s))$ satisfies the soundness property in the definition of an affine branching program: If $s \neq *$, the vertex $(v, s)$ is labeled by $s=\sigma_{v}(w)$ and by Poperty 2 of Lemma 4.3, $w \subseteq \sigma_{v}(w)$. If $s=*$, the vertex $(v, s)$ is labeled by $\{0,1\}^{n}$ and hence the soundness property is trivially satisfied.

\section{Proof of the Inductive Hypothesis:}

Next, we will prove the inductive hypothesis (Equation (1)), for $P_{j}$. We will define the random variable $U_{j} \in \mathcal{L}_{j}$ as follows:

As before, let $U_{j-1} \in \mathcal{L}_{j-1}$ be the random variable that satisfies the inductive hypothesis (Equation (11) ) for layer $j-1$ of $P_{j-1}$. Let $y_{j-1}$ be a random variable uniformly distributed over the subspace $w\left(U_{j-1}\right)$. Let $a \in_{R}\{0,1\}^{n}$. Let $b=a \cdot y_{j-1}$. Let $E=\left(U_{j-1}, V\right)$ be the edge labeled by $(a, b)$ outgoing $U_{j-1}$ in $P_{j-1}$. Thus, $V$ is a vertex in layer $j$ of $P_{j-1}$. As before, let $W=w(E)=w\left(U_{j-1}\right) \cap\left\{x^{\prime} \in\{0,1\}^{n}: a \cdot x^{\prime}=b\right\}$. As before, for a vertex $v$ in layer $j$ of $P_{j-1}$, let $\sigma_{v}: \mathcal{A}(n) \rightarrow \mathcal{A}(n)$ be the partial function whose existence is guaranteed by Lemma 4.3, when applied on the random variable $W_{v}=W \mid(V=v)$, and extend $\sigma_{v}: \mathcal{A}(n) \rightarrow \mathcal{A}(n)$ so that it outputs the special value $*$ on every element where it was previously undefined.

We define $U_{j}=\left(V, \sigma_{V}(W)\right) \in \mathcal{L}_{j}$. Let $y_{j}$ be a random variable uniformly distributed over the subspace $w\left(U_{j}\right)$, and let $V_{j}$ be the vertex in $\mathcal{L}_{j}$, reached by the computation-path of $P_{j}$. We need to prove that

$$
\left|\mathbb{P}_{V_{j}, x}-\mathbb{P}_{U_{j}, y_{j}}\right|_{1} \leq 2 j \cdot 2^{-\left(r-\frac{n}{2}\right)} .
$$


Let $y_{j}^{\prime}$ be a random variable uniformly distributed over the subspace $W$. Equation (目) follows by the following two equations and by the triangle inequality:

$$
\begin{gathered}
\left|\mathbb{P}_{U_{j}, y_{j}^{\prime}}-\mathbb{P}_{U_{j}, y_{j}}\right|_{1} \leq 2 \cdot 2^{-\left(r-\frac{n}{2}\right)} . \\
\left|\mathbb{P}_{V_{j}, x}-\mathbb{P}_{U_{j}, y_{j}^{\prime}}\right|_{1} \leq 2(j-1) \cdot 2^{-\left(r-\frac{n}{2}\right)} .
\end{gathered}
$$

Thus, it is sufficient to prove Equation (3) and Equation (4). We will start with Equation (3).

By Property 3 of Lemma 4.3, for every $v$ in layer $j$ of $P_{j-1}$, and every $s \in \operatorname{image}\left(\sigma_{v}\right) \backslash\{*\}$,

$$
\left|\underset{W \mid(V=v),\left(\sigma_{v}(W)=s\right)}{\mathbf{E}}\left[\mathcal{U}_{W}\right]-\mathcal{U}_{s}\right|_{1}<2^{-\left(r-\frac{n}{2}\right)} .
$$

By the definitions of $y_{j}^{\prime}$ and $U_{j}$,

$$
\underset{W \mid(V=v),\left(\sigma_{v}(W)=s\right)}{\mathbf{E}}\left[\mathcal{U}_{W}\right]=\underset{W \mid\left(U_{j}=(v, s)\right)}{\mathbf{E}}\left[\mathcal{U}_{W}\right]=\mathbb{P}_{y_{j}^{\prime} \mid\left(U_{j}=(v, s)\right)} .
$$

By the definition of $y_{j}$,

$$
\mathcal{U}_{s}=\mathbb{P}_{y_{j} \mid\left(U_{j}=(v, s)\right)}
$$

Hence

$$
\left|\mathbb{P}_{y_{j}^{\prime} \mid\left(U_{j}=(v, s)\right)}-\mathbb{P}_{y_{j} \mid\left(U_{j}=(v, s)\right)}\right|_{1}<2^{-\left(r-\frac{n}{2}\right)} .
$$

Taking expectation over $U_{j}$, and taking into account that, by Property 1 of Lemma 4.3 , for every $v, \operatorname{Pr}\left(\sigma_{v}(W)=*\right) \leq 2^{-2 n}$, we obtain

$$
\left|\mathbb{P}_{U_{j}, y_{j}^{\prime}}-\mathbb{P}_{U_{j}, y_{j}}\right|_{1}=\underset{U_{j}}{\mathbf{E}}\left|\mathbb{P}_{y_{j}^{\prime} \mid U_{j}}-\mathbb{P}_{y_{j} \mid U_{j}}\right|_{1}<2^{-\left(r-\frac{n}{2}\right)}+2^{-2 n}
$$

which proves Equation (3).

We will now prove Equation (4). Let $\mathcal{T}$ be the following probabilistic transformation from $\mathcal{L}_{j-1} \times\{0,1\}^{n}$ to $\mathcal{L}_{j} \times\{0,1\}^{n}$. Given $(u, z) \in \mathcal{L}_{j-1} \times\{0,1\}^{n}$, the transformation $\mathcal{T}$ chooses $a \in_{R}\{0,1\}^{n}$ and $b=a \cdot z$, and outputs $(V, z)$, where $V \in \mathcal{L}_{j}$ is the vertex obtained by following the edge labeled by $(a, b)$ outgoing $u$ in $P_{j}$.

By the definition of the computation-path, $\mathcal{T}\left(V_{j-1}, x\right)$ has the same distribution as $\left(V_{j}, x\right)$. By the definition of $U_{j}, y_{j}, y_{j}^{\prime}$, we have that $\mathcal{T}\left(U_{j-1}, y_{j-1}\right)$ has the same distribution as $\left(U_{j}, y_{j}^{\prime}\right)$. Hence, by the triangle inequality and the inductive hypothesis,

$$
\left|\mathbb{P}_{V_{j}, x}-\mathbb{P}_{U_{j}, y_{j}^{\prime}}\right|_{1}=\left|\mathbb{P}_{\mathcal{T}\left(V_{j-1}, x\right)}-\mathbb{P}_{\mathcal{T}\left(U_{j-1}, y_{j-1}\right)}\right|_{1} \leq\left|\mathbb{P}_{V_{j-1}, x}-\mathbb{P}_{U_{j-1}, y_{j-1}}\right|_{1} \leq 2(j-1) \cdot 2^{-\left(r-\frac{n}{2}\right)},
$$

which gives Equation (四).

Since, by induction, layers 0 to $j-1$ of $P_{j-1}$ form an affine branching program for parity learning, and since we already saw that all the edges between layer $j-1$ and layer $j$ of $P_{j}$ satisfy the soundness property in the definition of an affine branching program, we have that layers 0 to $j$ of $P_{j}$ form an affine branching program for parity learning. 


\section{$P$ is $\epsilon$-Accurate:}

We will now prove that the final branching program $P=P_{m}$, that we obtained, satisfies the requirements of the lemma. We already know that $P$ is an affine branching program for parity learning.

We will start by proving that $P$ is $\epsilon$-accurate. Let $0 \leq t \leq m$. Let $V_{t}$ be the vertex in layer $t$ of $P$, reached by the computation-path of $P$. Let $z_{t}$ be a random variable uniformly distributed over the subspace $w\left(V_{t}\right)$, We need to prove that,

$$
\left|\mathbb{P}_{V_{t}, x}-\mathbb{P}_{V_{t}, z_{t}}\right|_{1} \leq \epsilon .
$$

Recall that by the inductive hypothesis (Equation (四)), there exists a random variable $U_{t}$ over layer $t$ of $P$, such that, if $y_{t}$ is a random variable uniformly distributed over the subspace $w\left(U_{t}\right)$, then

$$
\left|\mathbb{P}_{V_{t}, x}-\mathbb{P}_{U_{t}, y_{t}}\right|_{1} \leq \frac{\epsilon}{2}
$$

and this also implies

$$
\left|\mathbb{P}_{V_{t}}-\mathbb{P}_{U_{t}}\right|_{1} \leq \frac{\epsilon}{2}
$$

By the last inequality and since for every $v$ in layer $t$ of $P$, it holds that $\mathbb{P}_{z_{t} \mid\left(V_{t}=v\right)}=\mathbb{P}_{y_{t} \mid\left(U_{t}=v\right)}$ (since they are both uniformly distributed over $w(v)$ ), we have

$$
\left|\mathbb{P}_{V_{t}, z_{t}}-\mathbb{P}_{U_{t}, y_{t}}\right|_{1}=\left|\mathbb{P}_{V_{t}}-\mathbb{P}_{U_{t}}\right|_{1} \leq \frac{\epsilon}{2}
$$

Equation (5) follows by Equation (6), Equation (7) and the triangle inequality.

\section{$P$ Satisfies the Additional Properties:}

We will now prove that $P$ satisfies the two additional properties claimed in the statement of the lemma. The first property holds since Property 14 of Lemma 4.3 ensures that for every vertex in layers 1 to $m$ of the branching program $B$, we obtain at most $4 n \cdot 2^{\sum_{i=0}^{n-k-1}\left(r-\frac{i}{2}\right)}$ vertices in the branching program $P$ that are labeled with affine subspaces of dimension $k$.

It remains to prove the second property. Let $V_{m}=(V, S)$ be the vertex in layer $m$ of $P$, reached by the computation-path of $P$. Note that $V_{m}$ is a random variable that depends on $x, a_{1}, \ldots, a_{m}$ (and recall that $x, a_{1}, \ldots, a_{m}$ are uniformly distributed over $\{0,1\}^{n}$, and for every $\left.t, b_{t}=a_{t} \cdot x\right)$.

Note that $V$ is the vertex in layer $m$ of $B$, reached by the computation-path of $B$ (on the same $\left.x, a_{1}, \ldots, a_{m}\right)$. This is true since $P$ simulates $B$. More precisely, by the construction, if on $x, a_{1}, \ldots, a_{m}$, the program $P$ reaches $(V, S)$, then, on the same $x, a_{1}, \ldots, a_{m}$, the program $B$ reaches $V$.

Since the success probability of $B$ is $\beta$,

$$
\operatorname{Pr}[x \in w(V)]=\beta,
$$

where $w(V)$ is the label of $V$ in $B$. Let $y_{m}$ be a random variable uniformly distributed over the subspace $w\left(V_{m}\right)$, where $w\left(V_{m}\right)$ is the label of $V_{m}$ in $P$. Since $P$ is $\epsilon$-accurate,

$$
\left|\mathbb{P}_{V, x}-\mathbb{P}_{V, y_{m}}\right|_{1} \leq\left|\mathbb{P}_{V, S, x}-\mathbb{P}_{V, S, y_{m}}\right|_{1}=\left|\mathbb{P}_{V_{m}, x}-\mathbb{P}_{V_{m}, y_{m}}\right|_{1} \leq \epsilon .
$$

Thus,

$$
\operatorname{Pr}\left[y_{m} \in w(V)\right] \geq \operatorname{Pr}[x \in w(V)]-\epsilon=\beta-\epsilon .
$$


Let $k>k^{\prime}$. Recall that $w(V)$ is of dimension $\leq k^{\prime}$. Thus, if $w\left(V_{m}\right)$ is of dimension $\geq k$, the (conditional) probability that $y_{m} \in w(V)$ is at most $2^{k^{\prime}-k}$. Thus,

$$
\beta-\epsilon \leq \operatorname{Pr}\left[y_{m} \in w(V)\right] \leq \operatorname{Pr}\left[\operatorname{dim}\left(w\left(V_{m}\right)\right)<k\right]+2^{k^{\prime}-k} .
$$

That is,

$$
\operatorname{Pr}\left[\operatorname{dim}\left(w\left(V_{m}\right)\right)<k\right] \geq \beta-\epsilon-2^{-\left(k-k^{\prime}\right)} .
$$

\section{Time-Space Lower Bounds for Parity Learning}

In this section, we will use Lemma 6.1 to prove Theorem 2, our main result. Recall that Theorem 2 is stronger than Theorem [1, and hence Theorem 1 follows as well. We start by a lemma that will be used, in the proof of Theorem 2, to obtain time-space lower bounds for affine branching programs.

Lemma 7.1. Let $k<n$. Let $P$ be a length $m$ affine branching program for parity learning (of size $n$ ), such that, for every vertex $u$ of $P, \operatorname{dim}(w(u)) \geq k$. Let $v$ be a vertex of $P$, such that, $\operatorname{dim}(w(v))=k$. Then, the probability that the computation-path of $P$ reaches $v$ is at most

$$
m^{n-k} \cdot 2^{\sum_{j=0}^{n-k-1}(n-2 k-j)} .
$$

Proof. Let $s$ be the vector space "orthogonal" to $w(v)$ in $\{0,1\}^{n}$. That is,

$$
s=\left\{a \in\{0,1\}^{n}: \exists b \in\{0,1\} \forall x^{\prime} \in w(v): a \cdot x^{\prime}=b\right\} .
$$

Let $V_{0}, \ldots, V_{m}$ be the vertices on the computation-path of $P$. Note that $V_{0}, \ldots, V_{m}$ are random variables that depend on $x, a_{1}, \ldots, a_{m}$. For every $0 \leq i \leq m$, let $S_{i}$ be the vector space "orthogonal" to $w\left(V_{i}\right)$ in $\{0,1\}^{n}$. That is,

$$
S_{i}=\left\{a \in\{0,1\}^{n}: \exists b \in\{0,1\} \forall x^{\prime} \in w\left(V_{i}\right): a \cdot x^{\prime}=b\right\} .
$$

By the soundness property in Definition 5.2 , for every $1 \leq i \leq m$,

$$
S_{i} \subseteq \operatorname{span}\left(S_{i-1} \cup a_{i}\right) .
$$

For every $0 \leq i \leq m$, let $Z_{i}=\operatorname{dim}\left(S_{i} \cap s\right)$. Note that $Z_{0}=0$, and by Equation (8), for every $1 \leq i \leq m, Z_{i} \leq Z_{i-1}+1$. If the computation-path of $P$ reaches $v$ then for some $1 \leq i \leq m, Z_{i}=n-k$. Thus, if the computation-path of $P$ reaches $v$, there exist $n-k$ indices $i_{1}<\ldots<i_{n-k} \in[m]$, such that, the following event, denoted by $E_{i_{1}, \ldots, i_{n-k}}$, occurs:

$$
E_{i_{1}, \ldots, i_{n-k}}=\bigwedge_{j \in[n-k]}\left(Z_{i_{j}-1}=j-1\right) \wedge\left(Z_{i_{j}}=j\right) .
$$

(In particular, $E_{i_{1}, \ldots, i_{n-k}}$ occurs if for every $j$, we take $i_{j}$ to be the first $i$ such that $Z_{i}=j$ ). We will bound the probability that the computation-path of $P$ reaches $v$, by bounding $\operatorname{Pr}\left[E_{i_{1}, \ldots, i_{n-k}}\right]$, and taking the union bound over (less than) $m^{n-k}$ possibilities for $i_{1}, \ldots, i_{n-k} \in[m]$. 
Fix $i_{1}<\ldots<i_{n-k} \in[m]$. For $r \in\{0, \ldots, n-k\}$, let

$$
E_{i_{1}, \ldots, i_{r}}=\bigwedge_{j \in[r]}\left(Z_{i_{j}-1}=j-1\right) \wedge\left(Z_{i_{j}}=j\right) .
$$

Thus,

$$
\operatorname{Pr}\left[E_{i_{1}, \ldots, i_{n-k}}\right]=\prod_{j \in[n-k]} \operatorname{Pr}\left[E_{i_{1}, \ldots, i_{j}} \mid E_{i_{1}, \ldots, i_{j-1}}\right] .
$$

We will show how to bound $\operatorname{Pr}\left[E_{i_{1}, \ldots, i_{j}} \mid E_{i_{1}, \ldots, i_{j-1}}\right]$.

$$
\begin{gathered}
\operatorname{Pr}\left[E_{i_{1}, \ldots, i_{j}} \mid E_{i_{1}, \ldots, i_{j-1}}\right]=\operatorname{Pr}\left[\left(Z_{i_{j}-1}=j-1\right) \wedge\left(Z_{i_{j}}=j\right) \mid E_{i_{1}, \ldots, i_{j-1}}\right] \\
\quad=\operatorname{Pr}\left[\left(Z_{i_{j}-1}=j-1\right) \wedge\left(Z_{i_{j}-1}<Z_{i_{j}}\right) \mid E_{i_{1}, \ldots, i_{j-1}}\right] \\
\leq \operatorname{Pr}\left[\left(Z_{i_{j}-1}<Z_{i_{j}}\right) \mid E_{i_{1}, \ldots, i_{j-1}} \wedge\left(Z_{i_{j}-1}=j-1\right)\right] .
\end{gathered}
$$

Note that the event $E_{i_{1}, \ldots, i_{j-1}} \wedge\left(Z_{i_{j}-1}=j-1\right)$ that we condition on, on the right hand side, depends only on $x, a_{1}, \ldots, a_{i_{j}-1}$. We will bound the probability for the event $\left(Z_{i_{j}-1}<Z_{i_{j}}\right)$, conditioned on any event that fixes $Z_{i_{j}-1}$ and depends only on $x, a_{1}, \ldots, a_{i_{j}-1}$.

More generally, fix $1 \leq i \leq m$, and let $E_{i}^{\prime}$ be the event $\left(Z_{i-1}<Z_{i}\right)$. Let $E^{\prime}$ be any event that fixes $Z_{i-1}$ and depends only on $x, a_{1}, \ldots, a_{i-1}$. Without loss of generality, we can assume that the event $E^{\prime}$ just fixes the values of $x, a_{1}, \ldots, a_{i-1}$. We will show how to bound $\operatorname{Pr}\left[E_{i}^{\prime} \mid E^{\prime}\right]$.

Thus, we fix $x, a_{1}, \ldots, a_{i-1}$ and we will bound $\operatorname{Pr}\left[E_{i}^{\prime}\right]$ (conditioned on $x, a_{1}, \ldots, a_{i-1}$ ). By Equation (8), if $E_{i}^{\prime}$ occurs then $\operatorname{dim}\left(S_{i-1} \cap s\right)<\operatorname{dim}\left(S_{i} \cap s\right) \leq \operatorname{dim}\left(\operatorname{span}\left(S_{i-1} \cup a_{i}\right) \cap s\right)$, and hence $S_{i-1} \cap s \subsetneq \operatorname{span}\left(S_{i-1} \cup a_{i}\right) \cap s$, which implies that there exists $a \in S_{i-1}$, such that, $a \oplus a_{i} \in s$. For every fixed $a \in S_{i-1}$, the event $a \oplus a_{i} \in s$ occurs with probability $2^{\operatorname{dim}(s)-n}=2^{(n-k)-n}=2^{-k}$ (since $a_{i}$ is uniformly distributed and independent of $\left.x, a_{1}, \ldots, a_{i-1}\right)$. We will bound the probability for $E_{i}^{\prime}$ by taking a union bound over all possibilities for $a$, but doing so we take into account that $a \in S_{i-1}$ satisfies $a \oplus a_{i} \in s$ if and only if every $a^{\prime} \in a \oplus\left(S_{i-1} \cap s\right)$ satisfies $a^{\prime} \oplus a_{i} \in s$. Thus, we can take a union bound over $2^{\operatorname{dim}\left(S_{i-1}\right)-Z_{i-1}} \leq 2^{n-k-Z_{i-1}}$ possibilities (where we assume that $Z_{i-1}$ is fixed). Hence, by the union bound

$$
\operatorname{Pr}\left[E_{i}^{\prime} \mid E^{\prime}\right] \leq 2^{n-k-Z_{i-1}} \cdot 2^{-k}=2^{n-2 k-Z_{i-1}} .
$$

Thus, in particular, by Equation (9),

$$
\operatorname{Pr}\left[E_{i_{1}, \ldots, i_{j}} \mid E_{i_{1}, \ldots, i_{j-1}}\right] \leq 2^{n-2 k-(j-1)} .
$$

Hence,

$$
\operatorname{Pr}\left[E_{i_{1}, \ldots, i_{n-k}}\right] \leq \prod_{j \in[n-k]} 2^{n-2 k-(j-1)}=2^{\sum_{j=0}^{n-k-1}(n-2 k-j)} .
$$

By the union bound, the probability that the computation-path of $P$ reaches $v$ is at most

$$
m^{n-k} \cdot 2^{\sum_{j=0}^{n-k-1}(n-2 k-j)} .
$$


Theorem 2. For any $c<\frac{1}{20}$, there exists $\alpha>0$, such that the following holds: Let $B$ be a branching program of length at most $2^{\alpha n}$ and width at most $2^{\text {cn }{ }^{2}}$ for parity learning (of size $n)$, such that, the output of $B$ is always an affine subspace of dimension $\leq \frac{3}{5} n$. Assume for simplicity and without loss of generality that all leaves of $B$ are in the last layer. Then, the success probability of $B$ (that is, the probability that $x$ is contained in the subspace that $B$ outputs) is at most $O\left(2^{-\alpha n}\right)$.

Proof. Let $0<\alpha<\frac{1}{5}$ be a sufficiently small constant (to be determined later on). Let $B$ be a branching program of length $m=2^{\alpha n}$ and width $d=2^{c n^{2}}$ for parity learning (of size $n$ ), such that, the output of $B$ is always an affine subspace of dimension $\leq \frac{3}{5} n$. Assume for simplicity and without loss of generality that all leaves of $B$ are in the last layer. Denote by $\beta$ the success probability of $B$.

Let $r=\left(\frac{1}{2}+2 \alpha\right) \cdot n$. Let $k=\frac{4}{5} n$. By Lemma 6.1, there exists a length $m$ affine branching program $P$ for parity learning (of size $n$ ), such that:

1. The number of vertices in $P$, that are labeled with an affine subspace of dimension $k$, is at most

$$
4 n \cdot 2^{\sum_{i=0}^{n-k-1}\left(r-\frac{i}{2}\right)} \cdot d m .
$$

2. The output of $P$ is an affine subspace of dimension $\leq k$, with probability of at least

$$
\beta-4 \cdot 2^{-\alpha n}-2^{-\frac{1}{5} n} \geq \beta-5 \cdot 2^{-\alpha n} .
$$

Assume without loss of generality that every vertex $u$ of $P$, such that $\operatorname{dim}(w(u))=k$, is a leaf. (Otherwise, we can just redefine $u$ to be a leaf by removing all the edges going out of it). Assume without loss of generality that for every vertex $u$ of $P, \operatorname{dim}(w(u)) \geq k$. (Otherwise, we can just remove $u$ as it is unreachable from the start vertex, since we defined all vertices labeled by subspaces of dimension $k$ to be leaves and since by the soundness property in Definition 5.2, the dimensions along the computation-path can only decrease by 1 in each step).

By Lemma 7.1, and by substituting the values of $m, d, k, r$, the probability that the computation-path of $P$ reaches some vertex that is labeled with an affine subspace of dimension $k$ is at most

$$
\begin{gathered}
\left(4 n \cdot 2^{\sum_{i=0}^{n-k-1}\left(r-\frac{i}{2}\right)} \cdot d m\right) \cdot\left(m^{n-k} \cdot 2^{\sum_{i=0}^{n-k-1}(n-2 k-i)}\right) \\
=4 n m \cdot 2^{c n^{2}} \cdot\left(2^{\sum_{i=0}^{n-k-1}\left(\frac{1}{2} n+2 \alpha n-\frac{i}{2}\right)}\right) \cdot\left(2^{\alpha n(n-k)} \cdot 2^{\sum_{i=0}^{n-k}\left(-\frac{3}{5} n-i\right)}\right) \\
=4 n m \cdot 2^{c n^{2}} \cdot 2^{(n-k)\left(3 \alpha n-\frac{1}{10} n\right)} \cdot\left(2^{\sum_{i=0}^{n-k-1}\left(-\frac{3}{2} i\right)}\right) \\
=4 n m \cdot 2^{c n^{2}} \cdot 2^{(n-k)\left(3 \alpha n-\frac{1}{10} n\right)} \cdot 2^{-\frac{3}{4}(n-k) \cdot(n-k-1)} \\
=4 n m \cdot 2^{c n^{2}} \cdot 2^{\frac{1}{5} n\left(3 \alpha n-\frac{1}{10} n-\frac{3}{20} n+\frac{3}{4}\right)} \\
=4 n m \cdot 2^{n^{2}\left(c+\frac{3}{5} \alpha-\frac{1}{20}+\frac{3}{20 n}\right)} .
\end{gathered}
$$

Thus, if $\alpha<\frac{5}{3}\left(\frac{1}{20}-c\right)$, this probability is at most $2^{-\Omega\left(n^{2}\right)}$, and hence,

$$
\beta-5 \cdot 2^{-\alpha n} \leq 2^{-\Omega\left(n^{2}\right)} .
$$

That is,

$$
\beta \leq O\left(2^{-\alpha n}\right)
$$




\section{References}

[A99a] Miklós Ajtai: Determinism versus Non-Determinism for Linear Time RAMs. STOC 1999: 632-641 3

[A99b] Miklós Ajtai: A Non-linear Time Lower Bound for Boolean Branching Programs. FOCS 1999: 60-70 3

[ADR02] Yonatan Aumann, Yan Zong Ding, Michael O. Rabin: Everlasting security in the bounded storage model. IEEE Transactions on Information Theory 48(6): 1668-1680 (2002) 田

[AR99] Yonatan Aumann, Michael O. Rabin: Information Theoretically Secure Communication in the Limited Storage Space Model. CRYPTO 1999: 65-79 1

[B86] David A. Mix Barrington: Bounded-Width Polynomial-Size Branching Programs Recognize Exactly Those Languages in NC1. J. Comput. Syst. Sci. 38(1): 150-164 (1989) (also in STOC 1986) 3

[BJS98] Paul Beame, T. S. Jayram, Michael E. Saks: Time-Space Tradeoffs for Branching Programs. J. Comput. Syst. Sci. (JCSS) 63(4):542-572 (2001) (also in FOCS 1998) 3

[BSSV00] Paul Beame, Michael E. Saks, Xiaodong Sun, Erik Vee: Time-space trade-off lower bounds for randomized computation of decision problems. J. ACM (JACM) 50(2):154-195 (2003) (also in FOCS 2000) 3

[CM97] Christian Cachin, Ueli M. Maurer: Unconditional Security Against MemoryBounded Adversaries. CRYPTO 1997: 292-306 田

[DM04] Stefan Dziembowski, Ueli M. Maurer: On Generating the Initial Key in the Bounded-Storage Model. EUROCRYPT 2004: 126-137 1

[F97] Lance Fortnow: Time-Space Tradeoffs for Satisfiability. J. Comput. Syst. Sci. 60(2): 337-353 (2000) (also in CCC 1997) 3

[FLvMV05] Lance Fortnow, Richard J. Lipton, Dieter van Melkebeek, Anastasios Viglas: Time-space lower bounds for satisfiability. J. ACM 52(6): 835-865 (2005) 3

[M92] Ueli M. Maurer: Conditionally-Perfect Secrecy and a Provably-Secure Randomized Cipher. J. Cryptology 5(1): 53-66 (1992) 团

[vM07] Dieter van Melkebeek: A Survey of Lower Bounds for Satisfiability and Related Problems. Foundations and Trends in Theoretical Computer Science, 2: 197-303, 2007. 3

[S14] Ohad Shamir: Fundamental Limits of Online and Distributed Algorithms for Statistical Learning and Estimation. NIPS 2014: 163-171 2 
[SVW15] Jacob Steinhardt, Gregory Valiant, Stefan Wager: Memory, Communication, and Statistical Queries. Electronic Colloquium on Computational Complexity (ECCC) 22: 126 (2015) 囵, 2, 6

[V03] Salil P. Vadhan: Constructing Locally Computable Extractors and Cryptosystems in the Bounded-Storage Model. J. Cryptology 17(1): 43-77 (2004) (also in Crypto 2003) 团

[W06] Ryan Williams: Inductive Time-Space Lower Bounds for Sat and Related Problems. Computational Complexity 15(4): 433-470 (2006) 国

[W07] Ryan Williams: Time-Space Tradeoffs for Counting NP Solutions Modulo Integers. IEEE Conference on Computational Complexity 2007: 70-82 目 